\title{
Neuropsychiatric Effects of Antiviral Drugs
}

Nicholas Zareifopoulos ${ }^{1}$, Maria Lagadinou ${ }^{2}$, Anastasia Karela ${ }^{2}$, Ourania Kyriakopoulou ${ }^{2}$, Dimitrios Velissaris 3,2

1. Department of Psychiatry, University of Patras, School of Health Sciences, Patras, GRC 2. Emergency Department, General University Hospital of Patras, Patras, GRC 3. Department of Internal Medicine, University of Patras, School of Health Sciences, Patras, GRC

Corresponding author: Nicholas Zareifopoulos, nizareifopoulos@gmail.com

\begin{abstract}
The adverse events of antiviral drugs are dose-dependent and often reversible. The nervous system is often affected and to date, many studies have been published regarding the central nervous system toxicity of antiviral agents. They may cause significant neuropsychiatric complications, which range from mild symptoms such as irritability and difficulty sleeping to severe complications such as depression, psychosis, and painful peripheral neuropathy, side effects which may necessitate discontinuation of treatment. The pathogenetic mechanisms may involve molecular targets common to other centrally active drugs, including human monoamine oxidase-A (MAO-A), serotonin receptors, gamma-aminobutyric acid (GABA) GABA-A receptors, 5-HT2A and 5-HT2C receptors and others. Notable examples include oseltamivir which may act as MAO inhibitor and efavirenz, which has an affinity for serotonin 5-HT2 and GABA-A receptors, the serotonin transporter, the MAO enzyme, and the vesicular monoamine transporter, with subjective effects which may be similar to those of the psychedelic hallucinogen lysergic acid diethylamide (LSD). Other antiviral drugs with prominent nervous system effects include nucleoside reverse transcriptase inhibitors, which are associated with the development of peripheral neuropathy after prolonged use (an effect strongly associated with older drugs which have since fallen into disfavor such as stavudine) and interferons, which may cause depression. Clinicians should be familiar with such adverse effects in order to recognise them promptly once they occur and manage them appropriately.
\end{abstract}

Categories: Internal Medicine, Psychiatry, Infectious Disease

Keywords: central nervous system, antiviral, efavirenz, peripheral neuropathy, monoamine oxidase

\section{Introduction And Background}

The adverse effects of antiviral drugs are dose-dependent and often reversible. The most common side effects include flu-like symptoms and hematologic abnormalities such as anemia and neutropenia. Moreover, some antiviral drugs cause significant neuropsychiatric complications, which range from irritability to severe depressive syndrome, including depression, cognitive impairment, and sleep disturbance.

Received 07/15/2020

Review began 07/16/2020 Review ended 07/21/2020 Published 08/03/2020

() Copyright 2020 Zareifopoulos et al. This is an open access article distributed under the terms of the Creative Commons Attribution License CC-BY 4.0., which permits unrestricted use, distribution, and reproduction in any medium, provided the original author and source are credited.
Generally, neuropsychiatric side effects are defined as new neurological or psychiatric symptoms that develop during treatment or worsening of preexisting neurological or psychiatric disorders. It may be difficult to determine whether the clinical phenotype can be attributed to the viral illness itself, the immune response to it, or the drugs used to treat it.

This paper reviews the neuropsychiatric effects of all available antiviral medications. We focus mostly on the mental health and central nervous system (CNS) effects of these medications but also discuss, in some cases, peripheral neurotoxicities such as neuropathy and myopathy. By being aware of the neuropsychiatric side effects of antivirals, clinicians are able to tailor treatment more effectively to individual patients.

\section{Review}

\section{Methods of literature search}

The initial literature search was conducted via the PubMed MEDLINE and Google Scholar databases. Data for each drug-class, as well as for notable individual agents, were searched separately using multiple search strings (e.g., ribavirin AND neurotoxicity, efavirenz psychiatric effects, efavirenz AND psychosis, acyclovir AND nervous system). The search retrieved a total of 7,289 citations, which were narrowed down to 236 after initial screening. The final review was based on 123 articles, of which 50 (the ones most likely to be of interest to practicing clinicians regardless of specialty) were referenced in the text. We included case reports, case series, and reviews regarding adverse neuropsychiatric antiviral agent effects in the report, as well as randomized trials and preclinical findings related to possible therapeutic applications of antivirals, with the intent of providing a balanced overview of the literature on neuropsychiatric effects of antiviral drug that may be relevant to clinicians regardless of specialty. Unavailable articles, articles not in English, and book chapters that were not peer-reviewed were excluded, as were preclinical studies focusing on possible mechanisms of action for neuropsychiatric adverse effects with minimal clinical relevance. Reports of the 
clinical trial or observational study findings that did not present data on the nervous system adverse effects of the drugs were also excluded. The initial screening was performed independently by all authors.

\section{Neuropsychiatric effects of antiviral therapy in influenza}

Oseltamivir and zanamivir are neuraminidase inhibitors, used for treating influenza by shortening the duration of the disease, relieving the symptoms and reducing the complications and transmission of influenza [1]. Neuraminidase inhibition prevents the virus from spreading within the respiratory tract and infecting new cells.

Results from studies in the USA, Spain, Japan, China and South Korea associate oseltamivir with many neuropsychiatric adverse events and abnormal behaviors such as delusions and perceptual disorders, delirium and delirium-like events, frightening episodes, abrupt anger, delirious speech, jumping or falling from a height, depressive episodes, mania, suicidal feelings [1, 2]. Oseltamivir phosphate (OP) is an ethyl ester pro-drug requiring ester hydrolysis for conversion to the active form of the neuraminidase inhibitor oseltamivir carboxylate (OC). Oseltamivir inhibits human monoamine oxidase-A (MAO-A), which is related to excitatory behaviors. Regarding acute and chronic psychotic reactions, receptors such as GABA-A, GABA$\mathrm{B}, \mathrm{N}$-methyl D-aspartate (NMDA), and $\mathrm{Na}+$, and $\mathrm{Ca} 2+$ channels are thought to be other candidates for investigation. Another study reported that oseltamivir sialylates a serum glycolipid that stimulates D2 dopaminergic receptor. This mechanism is related to abnormal behavior reported in some children taking oseltamivir. Unchanged oseltamivir phosphate is suggested to be related to central nervous system reactions such as acute behavioral change, occurring in the early phase of treatment [3].

In March 2007, the Japanese Ministry of Health, Labour and Welfare (MHLW) warned against the use of oseltamivir in children aged 10-19 years because of the possible cause of abnormal behavior. The U.S. Food and Drug Administration (FDA) added a warning to the oseltamivir label in 2006 to draw attention to the risk of neuropsychiatric adverse events (NPAEs). However, a causal association between oseltamivir use and abnormal behaviors or sudden death has not been established and a number of publications suggested that there is no evidence, nor plausible mechanism of action, to link oseltamivir with neuropsychiatric adverse events. They concluded that the risk of abnormal behavior was increased by influenza, and not by oseltamivir use [4]. As far as the use of zanamivir is concerned, no major neuropsychiatric adverse events have been reported.

\section{Neuropsychiatric effects of antiviral therapy in herpes and cytomegalovirus (CMV)}

Aciclovir, valacyclovir, famciclovir are medications used for the treatment of herpes infections. Since the introduction of acyclovir, in the early 1980s, neuropsychiatric adverse events have been related to the use of it. The most common neuropsychiatric adverse events, which are reported are tremor, visual and auditory hallucinations, confusion, and coma. Most cases have been associated with acute and chronic renal failure, severe illness, and the simultaneous use of other neurotoxic drugs [5]. However, there are cases with neuropsychiatric adverse events, related to acyclovir with normal renal function [6]. The neurotoxicity of acyclovir is usually described within the first 24 to 72 hours of treatment and there is complete recovery within two to seven days after treatment cessation.

Valacyclovir is an L-valyl ester and oral pro-drug of acyclovir converted to acyclovir and L-valine by firstpass intestinal and/or hepatic metabolism. At the highest rate (89\%) acyclovir is excreted in the urine. Valacyclovir is more efficiently absorbed and increases serum concentrations more rapidly than acyclovir and is effective with less frequent administration. The neuropsychiatric adverse events related to valacyclovir include confusion, hallucination, disturbances of consciousness, ataxia, dysarthria, death elusions, psychosis, and mania. Valacyclovir toxicity occurs within 72 hours of treatment and recovery after four days after the treatment is discontinued [7].

In many cases, with neuropsychiatric adverse events related to the use of both acyclovir and valacyclovir, high serum and cerebrospinal fluid (CSF) concentrations of 9-carboxymethoxymethylguanine (CMMG) have been described [7]. CMMG is an acyclovir metabolite. In renal failure, a great proportion of acyclovir is metabolized to 9-carboxymethoxymethylguanine (CMMG), probably through the action of alcohol dehydrogenase (ADH) and aldehyde-dehydrogenase (ALDH) [5].

Acute psychosis has been reported in an AIDS patient with mild renal dysfunction who was administered intravenous gancyclovir. The psychosis occurred after 15 days of administration. Cessation of the gancyclovir and administration of haloperidol resulted in recovery. Most patients with psychosis due to valacyclovir's structural analogs, acyclovir and gancyclovir, occur in elderly, immune-compromised patients with poor renal functioning, who receive the medication by the intravenous route [8].

Regarding famcyclovir use, no major neuropsychiatric adverse events are described. Headache (9-23\%), migraine (<3\%), and paresthesia (<3\%) are occasionally reported. Foscarnet (trisodium phosphonoformate), an investigational pyrophosphate analog is increasingly used to treat refractory cytomegalovirus retinitis 
and mucocutaneous herpes simplex virus infections in immunocompromised patients. Foscarnet has been reported to cause abnormalities in serum calcium and phosphate, including cases of fatal hypocalcemia. Neurological adverse events, related to the use of foscarnet are mainly attributed to hypocalcemia [9].

\section{Neuropsychiatric effects of antiviral therapy for chronic hepatitis B (CHB)}

Chronic hepatitis B requires long-term treatment with antiviral drugs, so side effect burden and its effects on treatment adherence may be as important as the efficacy of the drugs used. Antiviral agents indicated for the treatment of hepatitis B include lamivudine, telbivudine, and entecavir (classified as nucleoside analogues), and the nucleotide analogues adefovir dipivoxil, tenofovir disoproxil fumarate and tenofovir alafenamide [10].

Lamivudine and telbivudine have been associated with myopathy and peripheral neuropathy, with the incidence of these effects being similar across all age groups and both genders [10]. The proposed mechanism underlying this reaction is the depletion of mitochondrial DNA [11]. An interesting report of two cases of lamivudine induced dystonia has been reported, which responded to prompt administration of anticholinergic agents and did not recur after lamivudine discontinuation [12]. The clinical presentation was similar to the acute dystonic reactions associated with antipsychotic drugs, which are mediated by dopamine D2 receptor antagonism in the striatum. At present, there is no evidence that lamivudine interacts with dopamine receptors, so this could be regarded as an extremely rare idiosyncratic reaction.

In another study, three of six patients with lamivudine or telbivudineassociated myopathy had a complaint of numbness in the distal end of limbs, suggesting peripheral neuropathy. The presence of neuropathy was confirmed by the electrophysiological studies and nerve biopsies by the study team. Out of 3,500 patients who received telbivudine monotherapy in clinical trials, $10(0.28 \%)$ were reported to have peripheral neuropathy compared to nine of 48 (18.75\%) patients who received combination therapy of pegylated interferon and telbivudine. Moreover, patients receiving telbivudine treatment, suffer from weakness in upper and lower extremities [10]. Myopathy is characterized by creatine kinase (CK) elevation alongside muscle pain and weakness. CK elevations are among the welldescribed adverse effects of nucleoside analogues (NAs), but they are not specific for myopathy and may be associated with strenuous exercise and many other illnesses. In addition, fatigue and headache were reported as the most frequent adverse events associated with telbivudine use.

Entecavir is a highly selective guanosine nucleoside analogue, approved by the FDA at a dose of $0.5 \mathrm{mg}$ in treatment-naive and $1 \mathrm{mg} / \mathrm{d}$ in lamivudine-resistant CHB patients in 2005. Psychiatric adverse events are rare in the published reports. The most frequent neuropsychiatric adverse events in clinical trials were headache (17\%-23\%), fatigue (10\%-13\%), and dizziness (9\%), while 1-10\% of patients treated with entecavir suffer from insomnia. Entecavir-associated myopathy and peripheral neuropathy cases were very rarely reported in the literature. Patients receiving entecavir presenting with severe lactic acidosis, complain of weakness, reduced general physical condition, and impaired consciousness [10].

\section{Neuropsychiatric effects of antiviral therapy in hepatitis C}

The management of patients with hepatitis $\mathrm{C}$ is very complex. Psychiatric symptoms during antiviral therapy are reported in 30-40\% of chronic hepatitis $\mathrm{C}(\mathrm{CHC})$ patients treated for 6-12 months and are much more troublesome due to their insidious onset, unpredictable nature, and potentially serious consequences.

The combination of pegylated interferon and ribavirin was the mainstay of treatment for hepatitis $\mathrm{C}$ infection until the approval of direct-acting antivirals. The drugs were not well tolerated and psychiatric disturbances were a common adverse event during treatment. Interferon is notorious for its depressogenic potential and patients treated with it commonly experienced apathy, anhedonia, loss of motivation, and depressed mood [13]. The use of ribavirin in combination with either interferon or direct-acting antivirals may be associated with a greater incidence of psychiatric disturbances compared to monotherapy [14].

Depression is among the most common treatment-limiting side effects of treatment with the combination of interferon and ribavirin [13]. However, clinically significant neurological toxicity is reported in less than five percent of treated patients [15].

The recent approval of highly effective and well-tolerated direct-acting antiviral (DAA) regimens has dramatically changed the approach to hepatitis $\mathrm{C}$ virus (HCV) infection management. New antivirals have been developed that directly inhibit HCV, are associated with high sustained virologic response (SVR) rates and better patient tolerance [14].

There is a relative paucity of data on the adverse effects of DAAs due to their relatively recent approval. An initial increase in the Beck depression inventory score was reported in one study upon the initiation of DAA treatment, but the scores normalized by the end of the study period (12 weeks) [16]. The central nervous system effects of DAAs may be more pronounced in patients with liver cancer, as one study found an 
Currently available data show that new antivirals do not have specific neuropsychiatric side effects. For telaprevir, the most common "psychiatric" adverse events are fatigue and insomnia. However, depression was only evaluated in one trial, with an incidence of 20-22\% in all groups [18]. Regarding boceprevir, no specific additional psychiatric side effects could be observed [19]. Although two patients from different groups committed suicide, no specific information about depression has been given. Other adverse effects (AEs) included nausea, headache, and fatigue [20]. Ledipasvir was found to be associated with the occurrence of headache, insomnia, and asthenia [16].

Volpato et al. reported that some degree of neuropsychiatric impairment was observed in relation to treatment with sofosbuvir-based regimens in patients with cirrhosis, but not in post-liver transplanted patients, suggesting that the former may be more sensitive to mild sofosbuvir-based regimen neurotoxicity [17]. Specifically, trials of simeprevir/sofosbuvir demonstrate lower rates of insomnia and fatigue as compared with rates from boceprevir and telaprevir and suggested that these new agents may be better tolerated [16, 21]. Side effects such as weakness (30\%-59\%), headache (20\%-30\%), irritability (10\%-16\%), and depression (1\%) have been reported in relation to treatment with sofosbuvir and may suggest mild neurotoxicity [21]. In patients receiving daclatasvir/sofosbuvir with ribavirin, the most common treatment associated with neuropsychiatric side effects included irritability, insomnia, asthenia, and fatigue [22]. At the end of a course of treatment with a sofosbuvir (SOF)-based regimen, patients with cirrhosis exhibited an increase in extra-slow EEG activity and a slowing in their reaction times [17].

There is relatively little data regarding the association between anxiety and treatment with DAAs. One study reported an increased incidence of anxiety with combination therapy as compared with monotherapy [23]. Preliminary data from clinical studies as well as clinical experience suggest that neuropsychiatric adverse events are relatively rare and usually transient as a result of DAA treatment for hepatitis C, but further research is required in larger patient cohorts to provide definitive information.

\section{Neuropsychiatric effects of antiviral drugs in HIV}

Nucleoside Reverse Transcriptase Inhibitors (NRTIs)

Nucleoside reverse transcriptase inhibitors (NRTIs) have been associated with a number of adverse effects attributable to their interaction with human enzymes similar to their retroviral target [24]. These molecules are nucleoside analogues which lack a 3-OH group, which is necessary for phosphodiester bond formation and chain elongation. They are processed by the retroviral enzymes as regular nucleotides, but their incorporation into the newly synthesized DNA strand causes premature termination of transcription, preventing antiretroviral replication. In a similar manner, they affect human mitochondrial DNA polymerase $Y$ and telomerase, inhibiting replication of mitochondrial DNA and telomere elongation, respectively [25, 26]. Inhibition of mitochondrial DNA polymerase may underlie the association of these drugs with a chronic toxidrome reminiscent of inherited mitochondrial disorders, which include peripheral neuropathy, myopathy [27]. All drugs of this category have been associated with this side effect profile, though the strength of the association varies. Didanosine and stavudine have been more strongly associated with peripheral neuropathy, whereas zidovudine has more commonly been associated with myopathy and myelotoxicity. The pro-drug tenofovir alafenamide, which leads to greater concentrations of the active compound in lymphoid tissue compared to other cells, may be better tolerated than the initial formulation of tenofovir disiproxil fumarate as well as most other NRTIs, though it is not devoid of such side effects.

Zidovudine is the first drug approved for the treatment of HIV and was a vital component of a first-line treatment since the 1990s. It has been associated with peripheral neuropathy attributed to depletion of mitochondrial DNA by the mechanism that is common for all NRTIs. The risk of neuropathy seems to be dependent upon the total cumulative exposure to the drug, with individuals undergoing a prolonged course of treatment at high doses being at greater risk. The effect seems to be reversible, with gradual symptom improvement following zidovudine discontinuation [28]. In contemporary clinical settings, the incidence of peripheral neuropathy or myopathy during treatment with zidovudine could be managed by drug discontinuation, given the availability of alternative treatment options [29].

Tenofovir disiproxil fumarate is the first-line treatment for both HIV and hepatitis B virus (HBV) infection, due to its similar efficacy and significantly improved safety profile compared to other NRTIs. Peripheral neuropathy is less common with tenofovir compared to other NRTIs (especially stavudine), although it does occur. Eight percent of participants developed sensory neuropathy in a South African cohort of 120 treatment-naive individuals [30]. Psychiatric adverse effects appear to be infrequent with the use of tenofovir, although the risk may be greater when it is used in combination with efavirenz. A report of nine cases of neuropsychiatric complications arising following tenofovir initiation in individuals on efavirenz containing regimen suggests the need for further monitoring and extra caution when these two drugs are to be combined [31]. The risk of these adverse effects may be reduced by the use of the more selective pro-drug tenofovir alafenamide, though further research is warranted to determine whether the difference in the toxicity profile of the two formulations is clinically significant [32]. 
Efavirenz is a non-nucleoside reverse transcriptase inhibitor (NNRTI), and a common component of highly active antiretroviral therapy (HAART) used to treat HIV infection. It is commonly used in combination with two nucleoside reverse transcriptase inhibitors, and it is available in a fixed-dose combination with emtricitabine and tenofovir, but tablets containing only efavirenz (to be used in combinations with other antiretroviral drugs) are also widely available. In the developed world, it is no longer considered a first-line choice for HAART due to its psychiatric side effects, which may limit patient compliance. Since the drug was approved, it has been known to produce a variety of psychiatric issues, including sleep disturbances (insomnia, nightmares), psychotic symptomatology (visual hallucinations, derealization, depersonalization), and mood dysregulation (precipitation of mania upon initiation, depression with chronic use) $[33,34]$. Initially, the mechanism for these effects was unknown though it was obvious that they were dosedependent and that tolerance to the acute psychotropic effects of the drug developed a few weeks after treatment initiation [35]. Abuse of efavirenz has also been reported, primarily in South Africa where crushed tablets of efavirenz were smoked in a combination of drugs (including marijuana and heroin) called whoonga [36]. Recent evidence from in vitro and animal studies indicate that efavirenz is a potent psychotropic drug with significant affinity GABA-A receptors, 5-HT2A, and 5-HT2C receptors, while it also functions as a monoamine oxidase inhibitor and a dual serotonin/dopamine reuptake inhibitor [37]. Efavirenz may also deplete mitochondrial DNA via a mechanism similar to NRTIs, though the clinical relevance of this is overshadowed by the psychotropic side effects which occur immediately upon treatment initiation. Behavioral studies in mice indicate that it may partially substitute for LSD and methylenedioxymethamphetamine (MDMA), suggesting it has a similar effect. Neuropsychiatric adverse events, including sleep disturbances, depression, suicidality, and hallucinations have been reported in all clinical studies of efavirenz, are far more likely to occur with efavirenz compared with other antiretroviral drugs and limit regimen tolerability dramatically, being the most common reason for discontinuation.

Nevirapine is another non-nucleoside reverse transcriptase inhibitor, which, unlike efavirenz, has been infrequently associated with neuropsychiatric adverse events. A series of three case reports [38] of psychotic symptomatology and mania attributed to nevirapine have been published, but such events have been exceedingly rare and do not appear to be related to off-target interactions of the drug. Nevirapine has, however, been associated with hepatotoxicity and peripheral neuropathy, similar to nucleoside reverse transcriptase inhibitors, as evidenced by the findings of a cohort study of patients on HAART. It remains, however, unclear whether the mechanism (inhibition of mitochondrial DNA polymerase $\mathrm{Y}$ ) is the same [39].

Protease Inhibitors

Saquinavir was the first agent of this class to be approved in 1995 with ritonavir following in 1996. Protease inhibitors may be used as a component of HAART instead of a non-nucleoside reverse transcriptase inhibitor, whereas they may also be used alone for maintenance therapy once a sustained virologic response has been achieved. As a class, they are notorious for pharmacokinetic interactions as ritonavir is a potent inhibitor of CYP3A4, potentiating the effect of drugs dependent upon CYP3A4 metabolism for inactivation. They have been associated with a lipodystrophy syndrome characterized by insulin resistance, dyslipidemia, central adiposity, and an increased risk of cerebrovascular disease [40].

Prolonged use may contribute to the development of dementia by promoting the pathogenesis of cerebral small vessel disease via this mechanism. Abuse of ritonavir has also been reported, especially in combination with other drugs of abuse, which may be potentiated by CYP3A4 inhibitors [41, 42]. Protease inhibitors have poor blood-brain barrier (BBB) penetrance, so CNS adverse effects are unlikely. They have, however, been associated with peripheral neurotoxicity, including perioral paraesthesia, taste alteration, and hearing loss. These effects may be mediated by depletion of neurotrophic factors secreted by macrophages at sensory ganglia. Ritonavir at therapeutic doses has been strongly associated with such adverse effects, but these appear to be much less frequent with other protease inhibitors [43].

\section{Integrase Inhibitors}

Raltegravir was the first drug of this class to be approved in 2007, with elvitegravir and dolutegravir being approved in 2012 and 2013, respectively. They inhibit retroviral integrase, the enzyme which enables the integration of the viral DNA transcript (synthesized via reverse transcription) into the host cell genome. It is hypothesized that they may have an improved tolerability profile compared to reverse transcriptase inhibitors due to limited off-target interactions [44]. They are among the most well-tolerated antiretroviral agents, although they have also been associated with adverse psychiatric effects, especially dolutegravir.

Side effects which have been reported include insomnia, hallucinations, abnormal dreams, mood disturbances (mostly depression), fatigue, and confusion, similar to efavirenz. Integrase inhibitorcontaining regimens are, however, far less likely than efavirenz containing HAART regimens to be associated with neuropsychiatric side effects [45]. In prospective clinical trials [46] discontinuation due to such effects was low (> 1\%) [47], though in cohort studies which reflect actual clinical practice, they appear to lead to treatment discontinuation more frequently and may contribute to decreased treatment adherence [48]. The 
association between dolutegravir and psychiatric adverse events is stronger than for the other integrase inhibitors, though the reason for this remains unknown. No studies have been published providing insight into the mechanisms of integrase inhibitor-induced neuropsychiatric events. Sleep disturbances due to integrase inhibitors may be partially attenuated by advising patients to take the drugs during the day [49].

Entry and Fusion Inhibitors

Among the most recently approved antiretrovirals are maraviroc and enfuvirtide, two agents belonging to this class. They are used in conjunction with other components of HAART. They are not first-line agents (mostly reserved for salvage therapy) and appear to be better tolerated compared to other antiretroviral drugs. They are well tolerated and have not been associated with treatment-limiting neuropsychiatric adverse effects. Substitution of efavirenz with one of these drugs may reduce the risk of neuropsychiatric effects, whereas substitution of an NRTI by a fusion inhibitor may partially attenuate peripheral neuropathy and myopathy without compromising treatment efficacy [50]. Further research is, however, needed to determine whether these drugs would be similarly well tolerated if they were used at a rate similar to firstline antiretrovirals. A summary of the data presented in the article is available in the form of a table (Table 1).

\begin{tabular}{|c|c|c|c|c|}
\hline Drug class & $\begin{array}{l}\text { Common } \\
\text { neuropsychiatric side } \\
\text { effects }\end{array}$ & Mechanism of neurotoxicity & Clinical relevance & Notes \\
\hline $\begin{array}{l}\text { Neuramınıdase } \\
\text { inhibitors }\end{array}$ & $\begin{array}{l}\text { Irritability, psychosis, } \\
\text { mania, more commonly in } \\
\text { children }\end{array}$ & $\begin{array}{l}\text { Unclear; evidence regarding } \\
\text { MAO inhibition or } \\
\text { monoaminergic modulation } \\
\text { inconclusive }\end{array}$ & $\begin{array}{l}\text { Contraindicated in children }<12 \text { years } \\
\text { old. Use in individuals with a history } \\
\text { of psychiatric disturbances only if } \\
\text { benefits clearly outweigh risk. }\end{array}$ & $\begin{array}{l}\text { Relevant only for } \\
\text { oseltamivir. }\end{array}$ \\
\hline $\begin{array}{l}\text { Antiehrpetic } \\
\text { drugs } \\
\text { (acyclovir, } \\
\text { ganciclovir } \\
\text { and analogs) }\end{array}$ & $\begin{array}{l}\text { Hallucinations, confusion, } \\
\text { acute psychosis with } \\
\text { acute onset }\end{array}$ & $\begin{array}{l}\text { Accumulation of neurotoxic } \\
\text { metabolites which are normally } \\
\text { excreted in the urine } \\
\text { (carboxymethoxymethylguanine) }\end{array}$ & $\begin{array}{l}\text { Neurotoxicity more pronounced in } \\
\text { patients with renal failure. If } \\
\text { absolutely indicated in this } \\
\text { population, psychiatric disturbances } \\
\text { can be treated symptomatically. }\end{array}$ & \\
\hline Foscarnet & $\begin{array}{l}\text { Altered mental status, } \\
\text { perioral paresthesia }\end{array}$ & $\begin{array}{l}\text { Electrolyte disturbances, } \\
\text { particularly hypocalcemia }\end{array}$ & $\begin{array}{l}\text { Monitor and correct electrolyte } \\
\text { abnormalities. }\end{array}$ & \\
\hline Rıbavırın & $\begin{array}{l}\text { Depressed mood, } \\
\text { irritability, anxiety, sleep } \\
\text { disturbances, sexual } \\
\text { dysfunction - gradual } \\
\text { onset }\end{array}$ & Unclear & $\begin{array}{l}\text { (direct-acting antiviral agents } \\
\text { preferred) or, if not feasible, } \\
\text { psychiatric consultation, addition of } \\
\text { an antidepressant. }\end{array}$ & $\begin{array}{l}\text { Used in combination } \\
\text { with interferon or } \\
\text { direct-acting antivirals } \\
\text { - not clear whether } \\
\text { side effects are } \\
\text { attributable to ribavirin } \\
\text { alone. }\end{array}$ \\
\hline $\begin{array}{l}\text { antiviral } \\
\text { agents for } \\
\text { hepatitis C }\end{array}$ & $\begin{array}{l}\text { Depression, insomnia, } \\
\text { irritability, anxiety }\end{array}$ & Unclear & $\begin{array}{l}\text { Address symptoms if necessary } \\
\text { (psychotherapy, initiation of an } \\
\text { antidepressant). }\end{array}$ & $\begin{array}{l}\text { Boceprevir based } \\
\text { regimens may be more } \\
\text { prone to causing NPAE } \\
\text { compared to } \\
\text { sofosbuvir-based } \\
\text { regimens. Concurrent } \\
\text { ribavirin use may } \\
\text { exacerbated NPAEs. }\end{array}$ \\
\hline $\begin{array}{l}\text { Nucleoside / } \\
\text { Nucleotide } \\
\text { analogues }\end{array}$ & $\begin{array}{l}\text { Peripheral neuropathy } \\
\text { and myopathy. } \\
\text { Idiosyncratic manic or } \\
\text { psychotic reactions to } \\
\text { treatment initiation or } \\
\text { dose adjustment }\end{array}$ & $\begin{array}{l}\text { More pronounced with older } \\
\text { agents (stavudine, zidovudine) } \\
\text { compared to the ones currently } \\
\text { recommended (lamivudine, } \\
\text { tenofovir) }\end{array}$ & $\begin{array}{l}\text { Peripheral neuropathy is more } \\
\text { pronounced with increased duration } \\
\text { of exposure and higher dosage. } \\
\text { Consider discontinuing the offending } \\
\text { drug. For idiosyncratic reactions, } \\
\text { discontinue offending agent, treat } \\
\text { symptomatically. }\end{array}$ & $\begin{array}{l}\text { The mechanism for } \\
\text { idiosyncratic adverse } \\
\text { events (psychosis - } \\
\text { mania due to } \\
\text { zidovudine, dystonia } \\
\text { due to lamivudine) is } \\
\text { unclear. }\end{array}$ \\
\hline Efavirenz & $\begin{array}{l}\text { Hallucinations, mood } \\
\text { dysregulation, abnormal } \\
\text { dreams, abuse potential, } \\
\text { the effect may be similar } \\
\text { to psychedelic } \\
\text { hallucinogens - most } \\
\text { pronounced upon }\end{array}$ & $\begin{array}{l}\text { and dopamine reuptake } \\
\text { inhibition, MAO inhibition, } \\
\text { GABA-A receptor modulation }\end{array}$ & $\begin{array}{l}\text { NPAEs are very common, switching to } \\
\text { an alternative agent may lead to the } \\
\text { resolution of symptoms. If abnormal } \\
\text { dreams are prominent avoid dosing at } \\
\text { night. In patients with a psychiatric } \\
\text { history or substance abuse, }\end{array}$ & $\begin{array}{l}\text { likely present in all } \\
\text { patients to some } \\
\text { degree. }\end{array}$ \\
\hline
\end{tabular}




\section{Cureus}

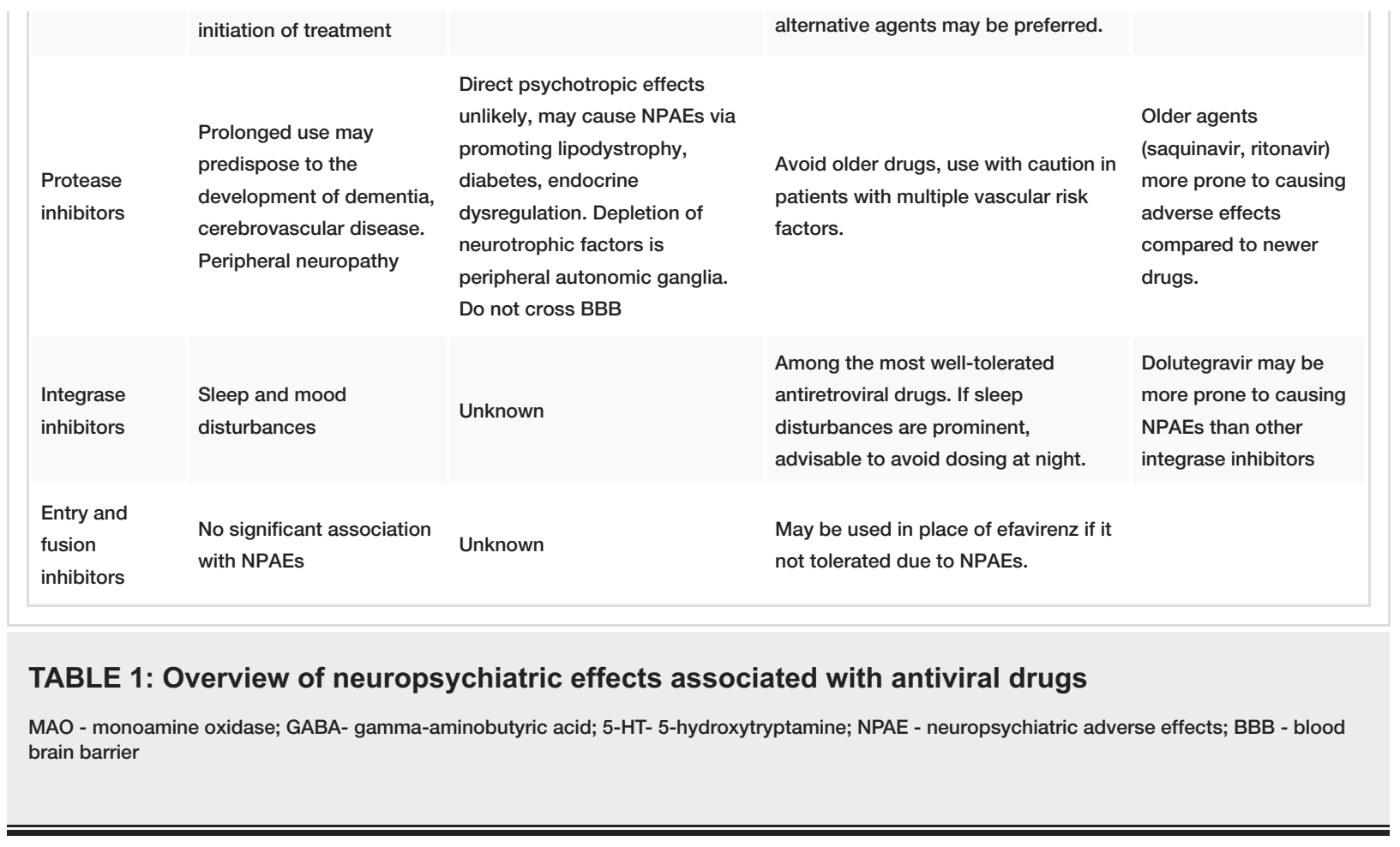

\section{Conclusions}

Neuropsychiatric effects of antiviral drugs are a common occurrence which complicates treatment. From the data presented in this article, it can be concluded that antivirals may affect the central nervous system, though for most drugs the reaction is idiosyncratic and the mechanisms are still unclear. Efavirenz has been associated with undesirable psychotropic effects similar to those observed with the use of psychedelic drugs, which are the most common reason for drug discontinuation. Further research is warranted in order to elucidate the mechanisms underlying the neuropsychiatric effects of antiviral drugs.

\section{Additional Information \\ Disclosures}

Conflicts of interest: In compliance with the ICMJE uniform disclosure form, all authors declare the following: Payment/services info: All authors have declared that no financial support was received from any organization for the submitted work. Financial relationships: All authors have declared that they have no financial relationships at present or within the previous three years with any organizations that might have an interest in the submitted work. Other relationships: All authors have declared that there are no other relationships or activities that could appear to have influenced the submitted work.

\section{References}

1. Chen R, Fang Z, Huang Y: Neuropsychiatric events in an adult patient with influenza a (H3N2) treated with oseltamivir (Tamiflu): a case report. BMC Infect Dis. 2019, 19:224. 10.1186/s12879-019-3827-4

2. Kang H-R, Lee E-K, Kim WJ, Shin J-Y: Risk of neuropsychiatric adverse events associated with the use of oseltamivir: a nationwide population-based case-crossover study. J Antimicrob Chemother. 2019, 74:453461. 10.1093/jac/dky445

3. Hama R, Bennett CL: The mechanisms of sudden-onset type adverse reactions to oseltamivir . Acta Neurol Scand. 2017, 135:148-160. 10.1111/ane.12629

4. Ueda N, Umetsu R, Abe J, et al.: Analysis of neuropsychiatric adverse events in patients treated with oseltamivir in spontaneous adverse event reports. Biol Pharm Bull. 2015, 38:1638-1644. 10.1248/bpb.b1500253

5. Hellden A, Lycke J, Vander T, Svensson J-O, Odar-Cederlof I, Stahle L: The aciclovir metabolite CMMG is detectable in the CSF of subjects with neuropsychiatric symptoms during aciclovir and valaciclovir treatment. J Antimicrob Chemother. 2006, 57:945-949. 10.1093/jac/dk1067

6. Skhiri H, Achour A, Skhiri S, et al.: Neuropsychiatric manifestations in a patient undergoing hemodialysis caused by treatment with oral acyclovir. Saudi J Kidney Dis Transpl. 2004, 15:50-52.

7. Asahi T, Tsutsui M, Wakasugi M, et al.: Valacyclovir neurotoxicity: clinical experience and review of the literature. Eur J Neurol. 2009, 16:457-460. 10.1111/j.1468-1331.2008.02527.x

8. Aslam SP, Carroll KA, Naz B, Alao AO: Valacyclovir-induced psychosis and manic symptoms in an adolescent young woman with genital herpes simplex. Psychosomatics. 2009, 50:293-296. 10.1176/appi.psy.50.3.293

9. Jacobson MA, Gambertoglio JG, Aweeka FT, Causey DM, Portale AA: Foscarnet-induced hypocalcemia and effects of foscarnet on calcium metabolism. J Clin Endocrinol Metab. 1991, 72:1130-1135. 10.1210/jcem-72- 
10. Kayaaslan B, Guner R: Adverse effects of oral antiviral therapy in chronic hepatitis B . World J Hepatol. 2017, 9:227-241. 10.4254/wjh.v9.i5.227

11. Kamara DA, Smith C, Ryom L, et al.: Longitudinal analysis of the associations between antiretroviral therapy, viraemia and immunosuppression with lipid levels: the D:A:D study. Antivir Ther. 2016, 21:495506. 10.3851/IMP3051

12. Song X, Hu Z, Zhang H: Acute dystonia induced by lamivudine . Clin Neuropharmacol. 2005, 28:193-194. 10.1097/01.wnf.0000172367.71932.61

13. Davoodi L, Masoum B, Moosazadeh M, Jafarpour H, Haghshenas MR, Mousavi T: Psychiatric side effects of pegylated interferon-alpha and ribavirin therapy in Iranian patients with chronic hepatitis C: a metaanalysis. Exp Ther Med. 2018, 16:971-978. 10.3892/etm.2018.6255

14. Sakamaki A, Kamimura K, Fukui N, et al.: A case report of psychiatric symptoms following direct-acting antiviral and ribavirin combination therapy for chronic hepatitis $\mathrm{C}$ in a patient with innate anxiety. BMC Gastroenterol. 2019, 19:85. 10.1186/s12876-019-1013-1

15. Fontana RJ: Neuropsychiatric toxicity of antiviral treatment in chronic hepatitis C . Dig Dis. 2000, 18:107116. $10.1159 / 000051384$

16. Takeda K, Noguchi R, Namisaki T, et al.: Efficacy and tolerability of interferon-free regimen for patients with genotype-1 HCV infection. Exp Ther Med. 2018, 16:2743-2750. 10.3892/etm.2018.6481

17. Volpato S, Montagnese S, Zanetto A, et al.: Neuropsychiatric performance and treatment of hepatitis C with direct-acting antivirals: a prospective study. BMJ Open Gastroenterol. 2017, 4:e000183. 10.1136/bmjgast2017-000183

18. Flamm SL, Pockros PJ, Bengtsson L, Friedman M: Patient characteristics, safety, and tolerability with telaprevir treatment for HCV in the clinic: a retrospective, multicenter study. J Clin Transl Hepatol. 2014, 2:65-73. 10.14218/JCTH.2014.00007

19. Manns MP, McCone JJ, Davis MN, et al.: Overall safety profile of boceprevir plus peginterferon alfa-2b and ribavirin in patients with chronic hepatitis $C$ genotype 1 : a combined analysis of 3 phase $2 / 3$ clinical trials. Liver Int. 2014, 34:707-719. 10.1111/liv.12300

20. Manns MP, Markova AA, Calle Serrano B, Cornberg M: Phase III results of Boceprevir in treatment naive patients with chronic hepatitis C genotype 1. Liver Int. 2012, 32:27-31. 10.1111/j.1478-3231.2011.02725.x

21. Yang Y-M, Choi EJ: Efficacy and safety outcomes of sofosbuvir-based treatment regimens for hepatitis C virus-infected patients with or without cirrhosis from phase III clinical trials. Ther Clin Risk Manag. 2017, 13:477-497. 10.2147/TCRM.S134818

22. Iriana S, Curry MP, Afdhal NH: Neurologic manifestations of hepatitis C virus infection. Clin Liver Dis. 2017, 21:535-542. 10.1016/j.cld.2017.03.008

23. Gallach M, Vergara M, da Costa JP, et al.: Impact of treatment with direct-acting antivirals on anxiety and depression in chronic hepatitis C. PLoS One. 2018, 13:0208112. 10.1371/journal.pone.0208112

24. Hirnschall G, Harries AD, Easterbrook PJ, Doherty MC, Ball A: The next generation of the World Health Organization's global antiretroviral guidance. J Int AIDS Soc. 2013, 16:18757. 10.7448/IAS.16.1.18757

25. Walker UA, Setzer B, Venhoff N: Increased long-term mitochondrial toxicity in combinations of nucleoside analogue reverse-transcriptase inhibitors. AIDS. 2002, 16:2165-2173. 10.1097/00002030-200211080-00009

26. Kakuda TN: Pharmacology of nucleoside and nucleotide reverse transcriptase inhibitor-induced mitochondrial toxicity. Clin Ther. 2000, 22:685-708. 10.1016/S0149-2918(00)90004-3

27. Reiss P, Casula M, de Ronde A, Weverling GJ, Goudsmit J, Lange JMA: Greater and more rapid depletion of mitochondrial DNA in blood of patients treated with dual (zidovudine+didanosine or zidovudine+zalcitabine) vs. single (zidovudine) nucleoside reverse transcriptase inhibitors. HIV Med. 2004, 5:11-14. 10.1111/j.1468-1293.2004.00178.x

28. Ndakala FN, Oyugi JO, Oluka MN, Kimani J, Jablonka A, Behrens GMN: Prevalent neuropathy in a cohort of HIV-infected Kenyan sex workers using antiretroviral drugs. Pan Afr Med J. 2016, 25:14. 10.11604/pamj.2016.25.14.9699

29. Agu KA, Oparah AC: Adverse drug reactions to antiretroviral therapy: Results from spontaneous reporting system in Nigeria. Perspect Clin Res. 2013, 4:117-124. 10.4103/2229-3485.111784

30. Pillay P, Wadley AL, Cherry CL, Karstaedt AS, Kamerman PR: Clinical diagnosis of sensory neuropathy in HIV patients treated with tenofovir: a 6-month follow-up study. J Peripher Nerv Syst. 2019, 24:304-313. 10.1111/jns.12349

31. Allavena C, Le Moal G, Michau C, Chiffoleau A, Raffi F: Neuropsychiatric adverse events after switching from an antiretroviral regimen containing efavirenz without tenofovir to an efavirenz regimen containing tenofovir: a report of nine cases. Antivir Ther. 2006, 11:263-265.

32. Gallant JE, Daar ES, Raffi F, et al.: Efficacy and safety of tenofovir alafenamide versus tenofovir disoproxil fumarate given as fixed-dose combinations containing emtricitabine as backbones for treatment of HIV-1 infection in virologically suppressed adults: a randomised, double-blind, activ. Lancet. 2016, 3:158-165. 10.1016/S2352-3018(16)00024-2

33. Kenedi CA, Goforth HW: A systematic review of the psychiatric side-effects of efavirenz . AIDS Behav. 2011, 15:1803-1818. 10.1007/s10461-011-9939-5

34. Clifford DB, Evans S, Yang Y, et al.: Impact of efavirenz on neuropsychological performance and symptoms in HIV-infected individuals. Ann Intern Med. 2005, 143:714-721. 10.7326/0003-4819-143-10-20051115000008

35. Juethner SN, Seyfried W, Aberg JA: Tolerance of efavirenz-induced central nervous system side effects in HIV-infected individuals with a history of substance abuse. HIV Clin Trials. 2003, 4:145-149. 10.1310/P7MJK2WD-T1FY-AU8L

36. Grelotti DJ, Closson EF, Smit JA, et al.: Whoonga: potential recreational use of HIV antiretroviral medication in South Africa. AIDS Behav. 2014, 18:511-518. 10.1007/s10461-013-0575-0

37. Gatch MB, Kozlenkov A, Huang R-Q, et al.: The HIV antiretroviral drug efavirenz has LSD-like properties . Neuropsychopharmacology. 2013, 38:2373-2384. 10.1038/npp.2013.135

38. Wise MEJ, Mistry K, Reid S: Drug points: Neuropsychiatric complications of nevirapine treatment . BMJ. 
2002, 324:879. 10.1136/bmj.324.7342.879

39. van Griensven J, Zachariah R, Rasschaert F, Mugabo J, Atte EF, Reid T: Stavudine- and nevirapine-related drug toxicity while on generic fixed-dose antiretroviral treatment: incidence, timing and risk factors in a three-year cohort in Kigali, Rwanda. Trans R Soc Trop Med Hyg. 2010, 104:148-153.

10.1016/j.trstmh.2009.07.009

40. Gupta S, Knight AG, Losso BY, Ingram DK, Keller JN, Bruce-Keller AJ: Brain injury caused by HIV protease inhibitors: role of lipodystrophy and insulin resistance. Antiviral Res. 2012, 95:19-29. 10.1016/. antiviral.2012.04.010

41. Cordova E, Cecchini D, Rodriguez C: Potential drug-drug interactions in HIV-perinatally infected adolescents on antiretroviral therapy in Buenos Aires, Argentina. J Int AIDS Soc. 2014, 17:19764. 10.7448/IAS.17.4.19764

42. Rough K, Dietrich J, Essien T, et al.: Whoonga and the abuse and diversion of antiretrovirals in Soweto, South Africa. AIDS Behav. 2014, 18:1378-1380. 10.1007/s10461-013-0683-x

43. Duval $\mathrm{X}$, Journot $\mathrm{V}$, Leport $\mathrm{C}$, et al.: Incidence of and risk factors for adverse drug reactions in a prospective cohort of HIV-infected adults initiating protease inhibitor-containing therapy. Clin Infect Dis. 2004, 39:248-255. 10.1086/422141

44. Hoffmann C, Llibre JM: Neuropsychiatric adverse events with dolutegravir and other integrase strand transfer inhibitors. AIDS Rev. 2019, 21:4-10. 10.24875/AIDSRev.19000023

45. Valcour VG, Spudich SS, Sailasuta N, et al.: Neurological response to cART vs. cART plus integrase inhibitor and CCR5 antagonist initiated during acute HIV. PLoS One. 2015, 10:0142600. 10.1371/journal.pone. 0142600

46. Curtis L, Nichols G, Stainsby C, et al.: Dolutegravir: clinical and laboratory safety in integrase inhibitornaive patients. HIV Clin Trials. 2014, 15:199-208. 10.1310/hct1505-199

47. Cohen C, Elion R, Ruane P, et al.: Randomized, phase 2 evaluation of two single-tablet regimens elvitegravir/cobicistat/emtricitabine/tenofovir disoproxil fumarate versus efavirenz/emtricitabine/tenofovir disoproxil fumarate for the initial treatment of HIV infection. AIDS. 2011, 25:7-12. 10.1097/QAD.0b013e328345766f

48. Hoffmann C, Welz T, Sabranski M, et al.: Higher rates of neuropsychiatric adverse events leading to dolutegravir discontinuation in women and older patients. HIV Med. 2017, 18:56-63. 10.1111/hiv.12468

49. Kim MJ, Kim S-W, Chang H-H, et al.: Comparison of antiretroviral regimens: adverse effects and tolerability failure that cause regimen switching. Infect Chemother. 2015, 47:231-238. 10.3947/ic.2015.47.4.231

50. Llibre JM, Rivero A, Rojas JF, et al.: Safety, efficacy and indications of prescription of maraviroc in clinical practice: Factors associated with clinical outcomes. Antiviral Res. 2015, 120:79-84. 10.1016/.antiviral.2015.05.001 